\title{
Relationship between the Subjective-Objective Oral Health Status and Oral Health Related Quality of Life in the Elderly
}

\author{
Ha-Young Youn, Min-Jeong Cho, Yoon-Sook Hwang ${ }^{1}$, and Kwang-Wook Koh ${ }^{2, \dagger}$ \\ Department of Preventive Dentistry, Kyungpook National University School of Dentistry, Daegu 41940, \\ ${ }^{1}$ Department of Dental Hygiene, Hanyang Women's University, Seoul 04763, \\ ${ }^{2}$ Department of Preventive Medicine, Kosin University College of Medicine, Busan 49267, Korea
}

The purpose of this study was to analyze the relationship between objective oral health status determined by dentists, self-perceived subjective oral health status, and oral health related quality of life (OHRQoL) in the elderly. The related factors affecting OHRQoL in the elderly were also surveyed. Four hundred and thirty elderly individuals who visited the three public health centers and four dental clinics in Busan were selected by convenience sampling. Twelve dental hygienists investigated the subjective oral health status and OHRQoL using the 14-item Oral Health Impact Profile (OHIP-14) and twentyone dentists examined the objective oral health status, including healthy remaining teeth, treated remaining teeth, functional remaining teeth, missing teeth, and non-treated missing teeth. Data were analyzed using SPSS ver. 12.0. OHRQLL was higher when oral and periodontal status was perceived as healthy, when there was no toothache, no interference in mastication, and when study subjects had the ability of food softening. It was also higher when study subjects had $\geq 20$ remaining teeth and $<9$ missing teeth, and were wearing denture. The related factors affecting OHRQoL of the elderly were the type of medical insurance, toothache, ability of food softening, perception of periodontal status, and the number of healthy remaining teeth. There was a significant relationship between the subjective-objective oral health status and OHRQoL in the elderly. A continuous oral health care system aimed at retaining $\geq 20$ healthy remaining teeth is needed to improve oral health and OHRQoL for the elderly, especially for the elderly receiving medical aid.

Key Words: 14-item Oral Health Impact Profile, Elderly, Oral health, Oral health related quality of life

\section{Introduction}

South Korea entered an era of 'aging society' in 2000 with $7.2 \%$ of the elderly population over 65 years of age, and it is predicted that the proportion will be $14.3 \%$ in 2018 , becoming an 'aged society,' and then $20.0 \%$ in 2025, a 'super aged society'1).

Oral health is closely related with systemic health conditions, including thyroid disease, arthritis, gastric ulcer, and cardiovascular disease ${ }^{2,3)}$, and poor oral health causes decline of masticatory function and tooth loss, which leads to limitation of interpersonal relations and social life, and causes psychosocial issues, resulting in a low quality of life ${ }^{4-6)}$. In particular, oral health is more important for health and quality of life in the elderly. An international study on quality of life found that promotion of oral health improved quality of life in the elderly, such as recovery of confidence and improvement in social relations ${ }^{7)}$. In a study in South Korea, the proportions of subjects aged $20 \sim 29$ years, $40 \sim 49$ years, and $\geq 60$ years who answered that oral health was the most important issue than any other health issues for pursuing quality of life were $19.8 \%, 41.7 \%$, and $64.5 \%$, respectively, indicating that oral health becomes more important with age ${ }^{8)}$.

In studies on relationship between oral health status and oral health related quality of life (OHRQoL) in the elderly, 
Chen and Hunter ${ }^{9)}$ reported numbers of decayed teeth, missing teeth, filled teeth, and type of dentist visit as factors affecting OHRQoL. McGrath and Bedi ${ }^{10)}$ reported that tooth loss without removable prosthesis declined OHRQoL. In addition, Wong and McMillan ${ }^{11)}$ reported that tooth loss and use of denture affected oral health related satisfaction of life. Studies in South Korea reported that tooth loss ${ }^{12)}$ and oral health level ${ }^{13,14)}$ of the elderly were correlated with the OHRQoL, and factors affecting OHRQoL of the elderly were number of missing teeth, subjective oral health status, and objective oral health status $^{15)}$.

As such, various studies comparatively analyzed direct and indirect relationships among factors, however, there has been limited data analyzing OHRQoL with subdividing remaining teeth in the elderly by preservation and prosthesis into healthy remaining teeth, treated remaining teeth, and functional remaining teeth, and with subdividing missing teeth by treatment into missing teeth and non-treated missing teeth. Thus, this study investigated self-perceived subjective oral health status and subdivided objective oral health status observed by specialists, in order to identify their relationships with OHRQoL. In addition, we aimed to provide fundamental data for improving oral health and OHRQoL in the elderly by identifying the related factors affecting OHRQoL.

\section{Materials and Methods}

\section{Subjects}

This study was conducted with 430 elderly who were 65 years or older and visited one of the three public health centers and four dental clinics in Busan Metropolitan City between July and September in 2016. Prior to participating, all participants were provided details of the study and written consent form. They understood the objective of the study and gave consent to participate. Finally, 376 questionnaires were used for analysis after excluding 54 questionnaires because of incomplete data. We obtained approval from the Institutional Review Board of Kosin University Gospel Hospital before initiation of the study (IRB no. 2016-07-015).

\section{Study methods}

The questionnaire consisted of 39 questions, including 8 questions for general characteristics, 5 questions for dental service use behavior, 5 questions for subjective oral health status, 7 questions for objective oral health status, and 14 questions for OHRQoL. Questions for subjective oral health status were about perception of oral status, toothache, interference in mastication, ability of food softening, and perception of periodontal status. For objective oral health status, dentists examined subjects using a dental mirror and a tweezer under the dental exam light, and recorded the number of remaining teeth as below 20 or at least 20 and the number of missing teeth as below 9 or at least $9^{16)}$. For the other questions, dental hygienists recorded the responses obtained via individual interviews.

Healthy remaining teeth referred to the number of permanent teeth except for the third molar, which included teeth that more than half of dental crown were visible, and teeth that were preserved or undergoing or with completed root canal treatment. However, tooth stumps or teeth to be extracted (e.g., teeth with 3 or higher degree of mobility) were excluded. Treated remaining teeth was the sum of numbers of healthy remaining teeth, cap crown, and abutment of fixed/removable denture, including teeth which is in the process for abutment formation. Functional remaining teeth were defined as the sum of numbers of treated remaining teeth, artificial teeth of fixed/removable denture and implants. Missing teeth refered to the number of teeth experiencing loss. Non-treated missing teeth was defined as the number of teeth that had not been treated after loss

For OHRQoL, subjects were questioned how often they were affected by oral issues during the recent one year period, using a simplified Oral Health Impact Profile-14 $(\mathrm{OHIP}-14)^{17)}$, and responses were scored as 0 for never, 1 for hardly ever, 2 for occasionally, 3 for very often, and 4 for fairly often. The highest score for each question was 4 points, and total score of all 14 questions was 56 points, in which a lower OHIP-14 meant a higher OHRQoL.

OHIP-14 in this study was analyzed by Cronbach's $\alpha$, in which confidence coefficients were 0.846 for functional limitation, 0.796 for physical pain, 0.856 for psycho- 
logical discomfort, 0.798 for physical disability, 0.850 for psychological disability, 0.818 for social disability, 0.850 for handicap, and 0.960 for OHIP-14.

\section{Analytical methods}

For general characteristics, subjective oral health status, objective oral health status, and OHRQoL, frequency and mean were analyzed by descriptive statistics. The difference in OHRQoL depending on general characteristics and oral health status was analyzed by t-test and ANOVA, and then Scheffe test was used for post-hoc analysis. To find out the related factors affecting OHRQoL, Models I and II were constructed, using factors with statistically significant difference as independent variables, followed by multiple regression analysis. Collected data were analyzed by using SPSS ver. 12.0 (SPSS Inc, Chicago, IL, USA), in which statistical significance level was set as 0.05 .

\section{Results}

\section{OHRQoL of subjects}

OHRQoL of subjects in seven subcategories was highest in social disability $(2.45 \pm 1.65)$, followed by psychological disability $(3.05 \pm 1.83)$, handicap $(3.17 \pm 1.85)$, physical disability (3.27 \pm 1.81$)$, physical pain (3.45 \pm 1.75$)$, functional limitation (3.50 \pm 2.03$)$, and psychological discomfort $(3.80 \pm 1.92)$. The score of OHIP-14 was $22.68 \pm$

Table 1. The OHRQoL of the Subjects

\begin{tabular}{lc}
\hline \multicolumn{1}{c}{ Variable } & Mean \pm SD \\
\hline Domains & \\
Functional limitation & $3.50 \pm 2.03$ \\
Physical pain & $3.45 \pm 1.75$ \\
Psychological discomfort & $3.80 \pm 1.92$ \\
Physical disability & $3.27 \pm 1.81$ \\
Psychological disability & $3.05 \pm 1.83$ \\
Social disability & $2.45 \pm 1.65$ \\
Handicap & $3.17 \pm 1.85$ \\
OHIP-14 & $22.68 \pm 11.28$ \\
\hline
\end{tabular}

OHIP items, ranging from 0 (never), 1 (hardly ever), 2 (occasionally), 3 (very often) to 4 (fairly often). Domains (2-items): $0 \sim 8$, OHIP-14 (14-items): $0 \sim 56$.

OHRQoL: oral health related quality of life, SD: standard deviation, OHIP-14: 14-item Oral Health Impact Profile.

\subsection{8 (Table 1)}

\section{OHRQoL depending on general characteristics of subjects}

In OHRQoL depending on general characteristics of the subjects, there were statistically significant differences depending on age, education level, presence or absence of spouse, type of medical insurance, and exercise. Regarding age as a variable, individuals aged $65 \sim 69$ years $(18.63 \pm 9.22)$ had the highst OHRQoL with statistically significant difference $(\mathrm{p}<0.05)$. For the variable of educational level, OHRQoL was highest in high school

Table 2. The OHRQoL according to General Characteristics $(n=376)$

\begin{tabular}{|c|c|c|c|}
\hline Characteristic & $\mathrm{n}(\%)$ & Mean \pm SD & p-value \\
\hline Gender & & & 0.974 \\
\hline Male & 185 (49.2) & $22.70 \pm 11.54$ & \\
\hline Female & $191(50.8)$ & $22.66 \pm 11.05$ & \\
\hline Age (y) & & & $<0.001$ \\
\hline $65 \sim 69$ & 179 (47.6) & $18.63 \pm 9.22^{\mathrm{b}}$ & \\
\hline $70 \sim 74$ & $83(22.1)$ & $25.57 \pm 10.46^{\mathrm{a}}$ & \\
\hline $75 \sim 79$ & $69(18.4)$ & $27.48 \pm 12.89^{\mathrm{a}}$ & \\
\hline$\geq 80$ & 45 (11.9) & $26.11 \pm 12.18^{\mathrm{a}}$ & \\
\hline Education & & & $<0.001$ \\
\hline No & $65(17.3)$ & $28.49 \pm 12.92^{\mathrm{a}}$ & \\
\hline Elementary school & $90(23.9)$ & $25.27 \pm 10.47^{\mathrm{a}, \mathrm{b}}$ & \\
\hline Middle school & $97(25.8)$ & $21.95 \pm 9.51^{\mathrm{b}, \mathrm{c}}$ & \\
\hline$\geq$ High school & $124(33.0)$ & $18.32 \pm 10.43^{\mathrm{c}}$ & \\
\hline Spouse & & & $<0.001$ \\
\hline Yes & $225(59.8)$ & $20.56 \pm 10.21$ & \\
\hline No & $151(40.2)$ & $25.83 \pm 12.07$ & \\
\hline Medical insurance & & & $<0.001$ \\
\hline Health insurance & $202(53.7)$ & $19.55 \pm 10.51$ & \\
\hline Medical aid & $174(46.3)$ & $26.30 \pm 11.08$ & \\
\hline Alcohol & & & 0.819 \\
\hline Yes & $128(34.0)$ & $22.49 \pm 11.60$ & \\
\hline No & $248(66.0)$ & $22.77 \pm 11.13$ & \\
\hline Smoking & & & 0.264 \\
\hline Yes & 86 (22.9) & $23.87 \pm 11.33$ & \\
\hline No & $290(77.1)$ & $22.32 \pm 11.26$ & \\
\hline Exercise & & & $<0.001$ \\
\hline Everyday & 63 (16.7) & $21.98 \pm 10.97^{\mathrm{b}}$ & \\
\hline Sometimes & $197(52.4)$ & $20.91 \pm 10.21^{\mathrm{b}}$ & \\
\hline No & $116(30.9)$ & $26.06 \pm 12.45^{\mathrm{a}}$ & \\
\hline
\end{tabular}

OHRQoL: oral health related quality of life, SD: standard deviation.

By independent t-test or one-way ANOVA.

${ }^{\mathrm{a} \sim \mathrm{c}}$ Different characters mean significant difference between groups by Scheffe test. 
graduates and higher $(18.32 \pm 10.43)$, followed by middle school graduates $(21.95 \pm 9.51)$, elementary school graduates $(25.27 \pm 10.47)$, and uneducated subjects $(28.49 \pm 12.92)(\mathrm{p}<0.05)$. Subjects with spouse $(20.56 \pm$ $10.21)$, health insurance policyholders $(19.55 \pm 10.51)$, and those who exercised sometimes $(20.91 \pm 10.21)$ showed higher OHRQoL ( $\mathrm{p}<0.05$, Table 2$)$.

\section{OHRQoL depending on oral health status of subjects}

OHRQoL depending on oral health status of the subjects was higher in those who perceived their oral status as healthy $(15.73 \pm 8.73)$, those who had no interference in mastication (15.95 \pm 8.30$)$, and those who were able to soften food $(15.13 \pm 7.06)(\mathrm{p}<0.05)$. In addition, OHRQoL became higher in those who had toothache less frequently and perceived periodontal status as healthier ( $p$ $<0.05$ ). OHRQoL was higher in those who had at least 20 healthy remaining teeth, treated remaining teeth, and functional remaining teeth $(\mathrm{p}<0.05)$, and had $<9$ missing teeth and non-treated missing teeth $(\mathrm{p}<0.05$, Table 3$)$.

\section{The related factors affecting OHRQoL}

The related factors affecting OHRQoL of subjects were the type of medical insurance $(\beta=0.12, p<0.05)$, toothache $(\beta=-0.30, p<0.05)$, ability of food softening $(\beta=$ $0.25, \mathrm{p}<0.05)$, perception of periodontal status $(\beta=-0.19$, $\mathrm{p}<0.05)$ and healthy remaining teeth $(\beta=-0.13, \mathrm{p}<0.05)$ in Model I. In Model II, influencing factors were the type of medical insurance $(\beta=0.12, p<0.05)$, toothache $(\beta=$ $-0.29, \mathrm{p}<0.05)$, ability of food softening $(\beta=0.24, \mathrm{p}<$ $0.05)$, perception of periodontal status $(\beta=-0.19, \mathrm{p}<$ $0.05)$, and healthy remaining teeth $(\beta=-0.13, \mathrm{p}<0.05)$. Explanatory powers of each model were $55.1 \%$ and $55.5 \%$, respectively (Table 4 ).

\section{Discussion}

Poor oral health has a negative impact on systemic health conditions ${ }^{2,3)}$, and restricts interpersonal relations and social life because of the decline in masticatory function and induction of tooth loss, leading to degradation of overall quality of life $\mathrm{e}^{4-6)}$. Therefore, it is necessary to promote oral health and OHRQoL of the elderly in order to improve their health and quality of life. Thus, this study investigated the relationship between oral

Table 3. The OHRQoL according to the Oral Health Status $(n=376)$

\begin{tabular}{|c|c|c|c|}
\hline Characteristic & $\mathrm{n}(\%)$ & mean \pm SD & p-value \\
\hline \multicolumn{4}{|l|}{ Subjective } \\
\hline \multicolumn{2}{|c|}{ Perception of oral status } & & $<0.001$ \\
\hline Not healthy & $287(76.3)$ & $24.83 \pm 11.12$ & \\
\hline Healthy & 89 (23.7) & $15.73 \pm 8.73$ & \\
\hline \multicolumn{2}{|l|}{ Toothache } & & $<0.001$ \\
\hline Often & $47(12.5)$ & $35.60 \pm 10.53^{\mathrm{a}}$ & \\
\hline Sometimes & $212(56.4)$ & $22.98 \pm 9.95^{\mathrm{b}}$ & \\
\hline Never & $117(31.1)$ & $16.95 \pm 9.30^{c}$ & \\
\hline \multicolumn{2}{|c|}{ Interference in mastication } & & $<0.001$ \\
\hline Yes & $227(60.4)$ & $37.09 \pm 10.80$ & \\
\hline No & 149 (39.6) & $15.95 \pm 8.30$ & \\
\hline \multicolumn{2}{|c|}{ Ability of food softening } & & $<0.001$ \\
\hline Yes & $148(39.4)$ & $15.13 \pm 7.06$ & \\
\hline No & $228(60.6)$ & $27.58 \pm 10.80$ & \\
\hline \multicolumn{2}{|c|}{ Perception of periodontal status } & & $<0.001$ \\
\hline Not healthy & $148(39.4)$ & $30.11 \pm 10.84^{\mathrm{a}}$ & \\
\hline Moderate & $200(53.2)$ & $18.70 \pm 8.63^{\mathrm{b}}$ & \\
\hline Healthy & $28(7.4)$ & $11.79 \pm 5.88^{\mathrm{c}}$ & \\
\hline \multicolumn{4}{|l|}{ Objective } \\
\hline \multicolumn{2}{|c|}{ Healthy remaining teeth } & & $<0.001$ \\
\hline$<20$ & $275(73.1)$ & $25.35 \pm 11.09$ & \\
\hline$\geq 20$ & $101(26.9)$ & $15.40 \pm 8.17$ & \\
\hline \multicolumn{2}{|c|}{ Treated remaining teeth } & & $<0.001$ \\
\hline$<20$ & $210(55.9)$ & $27.10 \pm 11.26$ & \\
\hline$\geq 20$ & $166(44.1)$ & $17.08 \pm 8.49$ & \\
\hline \multicolumn{2}{|c|}{ Functional remaining teeth } & & $<0.001$ \\
\hline$<20$ & $23(6.1)$ & $34.87 \pm 11.13$ & \\
\hline$\geq 20$ & $353(93.9)$ & $21.88 \pm 10.84$ & \\
\hline \multicolumn{2}{|l|}{ Missing teeth } & & $<0.001$ \\
\hline$<9$ & $165(43.9)$ & $17.23 \pm 8.63$ & \\
\hline$\geq 9$ & $211(56.1)$ & $26.94 \pm 11.29$ & \\
\hline \multicolumn{2}{|c|}{ Non-treated missing teeth } & & $<0.001$ \\
\hline$<9$ & $350(93.1)$ & $21.78 \pm 10.80$ & \\
\hline$\geq 9$ & $26(6.9)$ & $34.81 \pm 10.72$ & \\
\hline \multicolumn{2}{|c|}{ Experience of prosthesis } & & 0.967 \\
\hline Yes & $338(89.9)$ & $22.69 \pm 11.05$ & \\
\hline No & $38(10.1)$ & $22.61 \pm 13.29$ & \\
\hline \multicolumn{2}{|l|}{ Wearing denture } & & $<0.001$ \\
\hline Yes & $189(50.3)$ & $26.15 \pm 10.85$ & \\
\hline No & $187(49.7)$ & $19.17 \pm 10.62$ & \\
\hline
\end{tabular}

OHRQoL: oral health related quality of life, SD: standard deviation.

By independent t-test or one-way ANOVA.

${ }^{\mathrm{a} \sim \mathrm{c}}$ Different characters mean significant difference between groups by Scheffe test. 
Table 4. The Related Factors Affecting OHRQoL

\begin{tabular}{|c|c|c|c|c|}
\hline \multirow{2}{*}{ Variable } & \multicolumn{2}{|c|}{ Model I } & \multicolumn{2}{|c|}{ Model II } \\
\hline & $\beta(\mathrm{SE})$ & $\mathrm{p}$-value & $\beta(\mathrm{SE})$ & p-value \\
\hline \multicolumn{5}{|l|}{ General characteristics } \\
\hline Age & $0.01(0.48)$ & 0.771 & $0.01(0.48)$ & 0.868 \\
\hline Education & $-0.04(0.46)$ & 0.325 & $-0.04(0.46)$ & 0.400 \\
\hline Spouse & $0.02(0.95)$ & 0.604 & $0.03(0.97)$ & 0.511 \\
\hline Medical insurance & $0.12(1.01)$ & 0.006 & $0.12(1.03)$ & 0.009 \\
\hline Exercise & $0.07(0.61)$ & 0.056 & $0.06(0.61)$ & 0.093 \\
\hline \multicolumn{5}{|l|}{ Dental sevice use behavior } \\
\hline Type of dentist visit & & & $0.02(1.21)$ & 0.595 \\
\hline Experience of no dentist visit & & & $-0.06(0.94)$ & 0.130 \\
\hline Type of discomfort & & & $-0.01(0.54)$ & 0.791 \\
\hline Experience of illegal treatment & & & $-0.03(0.92)$ & 0.519 \\
\hline \multicolumn{5}{|l|}{ Subjective oral health status } \\
\hline Perception of oral status & $-0.02(1.15)$ & 0.639 & $-0.04(1.18)$ & 0.414 \\
\hline Toothache & $-0.30(0.75)$ & 0.000 & $-0.29(0.77)$ & $<0.001$ \\
\hline Interference in mastication & $-0.04(1.15)$ & 0.355 & $-0.05(1.11)$ & 0.298 \\
\hline Ability of food softening & $0.25(0.75)$ & 0.000 & $0.24(1.08)$ & $<0.001$ \\
\hline Perception of periodontal status & $-0.19(1.11)$ & 0.000 & $-0.19(0.91)$ & $<0.001$ \\
\hline \multicolumn{5}{|l|}{ Objective oral health status } \\
\hline Healthy remaining teeth & $-0.13(1.25)$ & 0.011 & $-0.13(1.27)$ & 0.009 \\
\hline Treated remaining teeth & $-0.04(3.82)$ & 0.805 & $-0.04(3.83)$ & 0.830 \\
\hline Functional remaining teeth & $-0.06(3.83)$ & 0.440 & $-0.07(3.84)$ & 0.398 \\
\hline Missing teeth & $0.03(3.59)$ & 0.841 & $0.03(3.60)$ & 0.859 \\
\hline Non-treated missing teeth & $0.07(3.66)$ & 0.382 & $0.06(3.68)$ & 0.451 \\
\hline Wearing denture & $-0.03(3.66)$ & 0.657 & $-0.02(1.39)$ & 0.709 \\
\hline
\end{tabular}

$\mathrm{R}^{2}=0.551, \mathrm{~F}=27.522, \mathrm{R}^{2}=0.555, \mathrm{~F}=22.132$. By multiple regression analysis.

OHRQoL: oral health related quality of life, SE: standard error.

health status and OHRQoL among elderly individuals over the age of 65 years and the related factors affecting their OHRQoL.

Within the total 56 points in OHIP-14, OHRQoL of the subjects was scored $22.68 \pm 11.28$ points showing slightly higher than the medium level. Of the subcategories, social disability was shown to be associated with the highest quality of life, whereas physical pain, functional limitation, and psychological discomfort was associated with relatively lower quality of life than the other categories, which was partially consistent with a report by Kim et al. $^{14)}$, which stated that physical pain and functional limitation were felt more prominently than others. In our study, psychological discomfort was associated with lower OHRQoL rather than functional limitation and physical pain. This seemed to be because there were many subjects who had restored teeth and recovered from pain by prosthesis treatment and dentures. Almost $90 \%$ and $50 \%$ of the subjects had prosthesis and dentures, respectively, while only $6.9 \%$ of the subjects had at least 9 non-treated missing teeth.

In terms of general characteristics of the subjects, OHRQoL was higher in younger age and was correlated with higher education level, which were consistent with a study by Steele et al. ${ }^{18)}$ showing negative relationship between OHRQoL and age, and a study by Lee ${ }^{19)}$ that showed a higher OHRQoL as education level became higher. Subjects with spouse had higher OHRQoL than those without spouse, which was consistent with the results of a study by Park et al. ${ }^{12)}$ indicating that the elderly cohabitating with spouse were less influenced by oral issues. Health insurance policyholders had higher OHRQoL than recipients of medical aid, which is similar to studies by Park et al. ${ }^{12)}$ and Lee and Park ${ }^{20)}$ that stated OHRQoL decreased with lower income. Subjects who exercised had higher OHRQoL, which was inconsistent 
with a report by Yom and $\operatorname{Han}^{21)}$ that stated exercise did not have an effect on OHRQoL.

Regarding subjective oral health status of the subjects, OHRQoL was higher in subjects who perceived their oral and periodontal status as healthier, had no toothache, had no interference in mastication, and were able to soften food. These results matched a report by Takata et al. ${ }^{22)}$, which stated that masticatory ability was a factor affecting OHRQoL, and a report by Jung and Shin ${ }^{23)}$ stating the level of oral pain was the most important factor. It was speculated that since subjects who had neither interference in mastication nor toothache feel less discomfort during food intake, hence perceive subjective oral status and periodontal status as healthy; therefore, their OHRQoL is higher.

As for objective oral health status, subjects who had at least 20 healthy remaining teeth, or treated remaining teeth, or functional remaining teeth, respectively showed higher OHRQoL, which was consistent with the results of study by Ryu et al. ${ }^{13)}$, which stated that subjects with at least 20 remaining teeth had higher OHRQoL. On the contrary, it was inconsistent with the results from a study by Takata et al. ${ }^{22)}$, which stated that there was no effect of remaining teeth on OHRQoL. In addition, subjects who had at least 20 healthy remaining teeth $(15.40 \pm 8.17)$ had relatively higher OHRQoL than those who had the same numbers of treated remaining teeth $(17.08 \pm 8.49)$ and functional remaining teeth $(21.88 \pm 10.84)$, indicating that healthy natural teeth without prosthesis treatment should have the highest positive effect on OHRQoL. Subjects with $<9$ missing teeth or $<9$ non-treated missing teeth showed higher OHRQoL. And subjects with at least 9 non-treated missing teeth $(34.81 \pm 10.72)$ had a lower quality of life than those with the same number of missing teeth (26.94 \pm 11.29$)$, indicating that the number of untreated teeth after loss had a substantially negative effect on OHRQoL.

To identify the related factors affecting OHRQoL of the subjects, multiple regression analysis was performed using the factors with statistically significant differences as independent variables and OHRQoL as dependent variable. In Model I, which considered only the general characteristics and oral health status, the related factors affecting OHRQoL were the type of medical insurance, toothache, ability of food softening, perception of periodontal status, and healthy remaining teeth, and the same factors were found in Model II which considered dental service use behavior additionally. These results were different from a report by Chen and Hunter ${ }^{9)}$, indicating that there is a relationship between the type of dentist visit and OHRQoL. In both models, OHRQoL was higher in health insurance policyholders, subjects who could soften food, those who had at least 20 healthy remaining teeth, those who felt toothache less frequently, and those who perceived their periodontal status as healthy as compared to others. The type of medical insurance may indicate economical level of the elderly, and economical imbalance in the elderly may also lead to imbalance of OHRQoL, therefore, it is necessary to manage oral health of the financially vulnerable elderly who receive medical aid. In addition, elderly with $<20$ remaining teeth could be in low-nutrition state owing to degradation of masticatory ability, suggesting that it should be closely related with systemic health condition ${ }^{16)}$. Hence, it is required to provide a systematic and continuous oral health care for the elderly.

To improve oral health and OHRQoL in the elderly, the results of the present study suggested that a continuous oral health care system for the elderly should be established with the aim of retaining at least 20 healthy remaining teeth while eliminating prosthesis treatment via prevention and early detection of oral diseases. Medical insurance should cover prosthesis treatment to restore functions of the missing teeth without leaving them untreated, or a prosthesis support system should be established for the elderly receiving medical aid.

There are some limitations of this study. First, subjects were recruited by convenience sampling in a city of South Korea; therefore, the results may not be generalized to the entire elderly population of South Korea. In addition, the elderly who stayed at home because of difficulties in mobility, and the elderly in facilities were not included, which limited the representativeness of the subjects of this study. To minimize deviations caused by oral examinations of many dentists, they should be complemented by single dentist investigator or several dentists thoroughly 
trained. Additionally, the study using OHIP-14 should be supplemented by using other tools in parallel. Despite these limitations, the results of this study may be partially accepted because the results of this study were mostly consistent with the previous studies.

\section{References}

1. KOSIS: Population projections. Retrieved July 14, 2017, from: http://kosis.kr/statHtml/statHtml.do?orgId=101\&tblId =DT_1BPA002\&conn_path=I2.xls(2016).

2. Bahekar AA, Singh S, Saha S, Molnar J, Arora R: The prevalence and incidence of coronary heart disease is significantly increased in periodontitis: a meta-analysis. Am Heart J 154: 830-837, 2007.

3. Molloy J, Wolff LF, Lopez-Guzman A, Hodges JS: The association of periodontal disease parameters with systemic medical conditions and tobacco use. J Clin Periodontol 31: 625-632, 2004.

4. Meeuwissen JH, van Waas MA, Meeuwissen R, Käyser AF, van't Hof MA, Kalk W: Satisfaction with reduced dentitions in elderly people. J Oral Rehabil 22: 397-401, 1995.

5. Gilbert GH, Foerster U, Duncan RP: Satisfaction with chewing ability in a diverse sample of dentate adults. J Oral Rehabil 25: 15-27, 1998

6. McGrath C, Bedi R: Can dental attendance improve quality of life? Br Dent J 190: 262-265, 2001.

7. Shtereva N: Aging and oral health related to quality of life in geriatric patients. Rejuvenation Res 9: 355-357, 2006.

8. Choi JS, Jung SH: Policy planning for oral health promotion. Korea Institute for Health and Social Affairs, Seoul, p.58, 2000.

9. Chen MS, Hunter P: Oral health and quality of life in New Zealand: a social perspective. Soc Sci Med 43: 1213-1222, 1996.

10. McGrath C, Bedi R: Can dentures improve the quality of life of those who have experienced considerable tooth loss? J Dent 29: 243-246, 2001.
11. Wong MC, McMillan AS: Tooth loss, denture wearing and oral health-related quality of life in elderly Chinese people. Community Dent Health 22: 156-161, 2005.

12. Park JH, Jeong SH, Lee GR, Song KB: The impact of tooth loss on oral health related quality of life among the elderly in Seongju, Korea. J Korean Acad Oral Health 32: 63-74, 2008.

13. Ryu KJ, Lee TY, Kim KY: A study on oral health-related quality of life of among elderly in metropolis. J Korean Soc Dent Hyg 9: 1-16, 2009.

14. Kim MH, Kim KW, Lee KS: Association between oral health and oral health-related quality of life among the elderly. J Dent Hyg Sci 14: 488-494, 2014.

15. Kim ES: Review of oral health-related quality of life using the oral health impact profile. J Korean Soc Dent Hyg 15: 13-22, 2013.

16. Budtz-Jørgensen E, Chung JP, Rapin CH: Nutrition and oral health. Best Pract Res Clin Gastroenterol 15: 885-896, 2001.

17. Lee MS, Kim SH, Yang JS, Oh JS, Kim DK: Validity and reliability of the oral health impact profile in elderly Korean 65+. J Korean Acad Oral Health 29: 210-221, 2005.

18. Steele JG, Sanders AE, Slade GD, et al.: How do age and tooth loss affect oral health impacts and quality of life? A study comparing two national samples. Community Dent Oral Epidemiol 32: 107-114, 2004.

19. Lee GR: A study of oral health impact profile 14 among the elderly in rural area. J Dent Hyg Sci 10: 109-116, 2010.

20. Lee HO, Park JY: Relationship between oral health behavior and happiness index in elderly people. J Dent Hyg Sci 16: 415-423, 2016.

21. Yom YH, Han JH: Factors associated with oral health related-quality of life in the elderly persons: applying Andersen's model. Korean J Fundam Nurs 21: 18-28, 2014.

22. Takata Y, Ansai T, Awano S, et al.: Chewing ability and quality of life in an 80-year-old population. J Oral Rehabil 33: 330-334, 2006.

23. Jung YM, Shin DS: Oral health, nutrition, and oral health-related quality of life among Korean older adults. J Gerontol Nurs 34: 28-35, 2008. 